\title{
ON-ROAD EVALUATION OF DRIVER CAPABILITY: A MEDICAL RECORD REVIEW OF THE ADAPTIVE DRIVING PROGRAM
}

\author{
Nahom Beyene, Amy Lane, Rosemarie Cooper \& Rory Cooper \\ Department of Rehabilitation Science and Technology, University of Pittsburgh \\ Pittsburgh, Pennsylvania, USA \\ Email:nmb32@pitt.edu
}

\begin{abstract}
Summary: a) The purpose of the present study was to illustrate how driver capability could be measured based on the presence of assistance during on-road evaluation. As an objective, this study explored the potential of a new method to measure declines in driver independence (steering/braking assistance) and safety (driving cues) for driver fitness determinations. b) A study at the Adaptive Driving Program (ADP) was conducted through a medical record review of 132 clients served in 2009. Following creation of an enumerated list of unique errors committed in baseline driving sessions, follow-up analysis focused on the association between assistance during on-road evaluation and case outcomes. The analysis also involved associations between assistance and five classes of errors reported among all clients. c) Findings showed that the proposed measures of driver independence and safety were associated with $90 \%$ of clients that did not pass on-road evaluation and a majority of errors related to tracking vehicle position within a lane. Though documented assistance showed low association to four out of five classes of errors, the potential for detection of these assistedevents may be $60-80 \%$ of all errors in each class except for lane changes.
\end{abstract}

\section{OBJECTIVES}

Nearly all driving evaluations involve a report that either enumerates or scores performance based on errors committed under observation. The challenge of driver capability measurement relates to the complexity of the many factors which contribute to a "Swiss cheese" model of risk (Sheridan, 2008), where potentially negligible, individual errors (holes) line up undetected to allow crash risk to pass through protections and become a crash realized. In each scenario of a committed driving error, factors such as the driving maneuver, posted traffic signals and signs, road obstacles, road quality, weather conditions, presence of other road users, and pedestrians are among the many issues complicating a direct question: Is my client fit to drive based on today's demonstration of driver capability?

Physicians face great difficulty when advising concerns with driving, especially when they are voiced from family members and friends who are unable to reach an agreement with the license holder (the patient). In order to ease the tension between physicians and their patients, a (Certified) Driver Rehabilitation Specialist can provide additional evidence to assist in the determination of fitness to drive. Physicians surveyed in a study (Jang et al., 2007) had reported that $75 \%$ viewed the act of reporting to present a conflict of interest in the physician-patient relationship, and $45 \%$ were not confident with the responsibility of reporting. The AMA ADReS Guidelines (Carr, 2010) define a standard for primary care physicians to assess driving related skills for patients who are known to be driving. 
Any recommendations for driver cessation should be based on the dominant scenario of driver error as the causal basis for a potential collision. The specific nuance of each crash condition defines a myriad list of unique driving errors. Yet, driver error can be documented via measures of independence and safety in vehicle operations and driving decisions. The purpose of the present study is to illustrate how driver capability could be measured based on the presence of assistance during on-road evaluation. Two objectives motivate this study of a new method to measure driver independence and safety in determination of driver capability:

1. To investigate frequency and hierarchy of driving errors as associated with assistance in a driving session including steering/braking assistance or driving cues

2. To identify limitations of the association between unique driving errors and assistance during on-road evaluation

\section{METHODS}

A review of the Adaptive Driving Program (ADP) occurred through a medical record review following approval by the Institutional Review Board at the University of Pittsburgh. The inclusion criteria for review of client records required that the initial visit took place in 2009. In this way, clients served in 2009 with initial intake in 2008 were excluded. Any clients pending case closures at the end of 2009 were tracked into the following year in order to obtain the outcomes of their on-road evaluation and case resolution following training whenever applicable. All clients served during the review period received evaluations by the same Certified Driver Rehabilitation Specialist (CDRS).

\section{Digitization Protocol}

Each client's case records were digitized using raw data recording, and sensitive data was omitted where HIPAA identifiers were documented. A single coder reviewed the findings and recommendations from on-road evaluations for any legible documentation of assistance by the CDRS. Any reported assistance with driving received the label of a "cue" or "assist" to the client during the baseline driving evaluation. For the purpose of clarity, the baseline driving evaluation occurred as part of a comprehensive evaluation. The comprehensive evaluation took place on the occasion of the first session with a client and entailed a pre-driver's (clinical) assessment (unless performed by a third-party), and concluded with on-road driving evaluation. Records for training and the associated documents from those follow up client sessions were also digitized, but the records from those sessions were beyond the scope of the present analysis.

\section{Content Analysis}

The primary analysis produced an enumerated list of unique driving errors committed by all clients who received a comprehensive evaluation from the ADP. Along with the list, frequency counts demonstrated how many clients committed each specific error, summary class assignments joined similar error types, entry item coding maintained the context in which the items were observed, and phrases indicating assisted driving events recorded whether the assistance was implied (found in writing or evident in the words used to document an error) or potential (possible in the absence of explicit documentation). 
Based on the enumerated list, our secondary analysis involved the comparison of errors possibly involving assistance versus the outcome of on-road evaluations with implied (documented) assistance listed in their case report. The coder determined percentages by summing the number of assisted-events indicated within a class of errors and dividing by the total number of unique errors attributed to the class. In this way, the percentage of cases involving assistance also split the value among two groups: those that "did pass” and others who "did not pass."

\section{RESULTS}

The outcomes from on-the road driving resulted in pass (48\%), training with vehicle modifications (35.5\%), remedial training (14\%), and fail (2.5\%). Recommendations from the ADP were for 101 clients (82.8\%) to continue or commence driving, while 21 clients (17.2\%) failed to resume or begin driving. The client base included people of all ages (legal for driving) and several categories of disability (brain injury, stroke, neurological, multiple sclerosis, spinal cord injury, cognitive, amputee, etc.), but the vehicle modification training services only reflected low-tech solutions such as hand-controls or a left-foot accelerator.

An enumerated list of unique errors committed reflected two contexts based on locations where driving errors occur: road segments and road crossings (intersections). Table 1 shows three classes of errors that occurred on road segments traveled by 122 clients of the ADP. Tracking is a term used to indicate an effort to maintain stable positioning within a lane. The merge and lane change lists of errors involve any intentional departure from a lane on a road segment.

Table 1. Errors Associated with Driving on Road Segments

\begin{tabular}{|c|c|c|c|c|c|c|}
\hline Class & Total & Frequency & Errors & Context & \multicolumn{2}{|c|}{ Potential } \\
\hline \multirow{19}{*}{ Tracking } & \multirow{19}{*}{101} & 31 & too close to side of lane ---CUE & steering & $\mathbf{x}$ & \\
\hline & & 15 & oriented to keep hand on spinner knob ---CUE & steering & $\mathbf{x}$ & \\
\hline & & 12 & tends to drive under speed limit & speed control & $\mathbf{x}$ & \\
\hline & & 10 & would exceed speeding limit & speed control & $\mathbf{x}$ & $\mathbf{x}$ \\
\hline & & 5 & required assistance at times---ASSIST & steering & & $\mathbf{x}$ \\
\hline & & 5 & poor lane position & highway driving & $\mathbf{x}$ & $\mathbf{x}$ \\
\hline & & 3 & speed variable throughout session & speed control & & \\
\hline & & 3 & simultaneously gas and brake (pedal strike errors) & speed control & & \\
\hline & & 3 & oriented to proper vehicle spacing ---CUE & steering & $\mathbf{x}$ & \\
\hline & & 2 & unsafe hand positioning & steering & & \\
\hline & & 2 & vehicle spacing not tested & steering & & \\
\hline & & 2 & too slow, caused traffic to pass on right & highway driving & $\mathbf{x}$ & \\
\hline & & 2 & poor speed matching to flow of traffic & highway driving & & \\
\hline & & 1 & slow to react to lane position errors & steering & $\mathbf{x}$ & $\mathbf{x}$ \\
\hline & & 1 & unaware of lane departure & steering & $\mathbf{x}$ & \\
\hline & & 1 & dismissive of steering problem & steering & $\mathbf{x}$ & \\
\hline & & 1 & became nervous & highway driving & & \\
\hline & & 1 & not tested & highway driving & & \\
\hline & & 1 & just nervous & decision making & & \\
\hline \multirow{3}{*}{ Merge } & \multirow{3}{*}{8} & 5 & need to work on entry and exit & highway driving & $\mathbf{x}$ & $\mathbf{x}$ \\
\hline & & 2 & overyielding for merge of lane change & steering & & \\
\hline & & 1 & unnecessary stop on exit for merge & highway driving & & \\
\hline \multirow{6}{*}{ Lane Change } & \multirow{6}{*}{12} & 6 & lane change by mirrors only & rt and left turn & & \\
\hline & & 2 & lane change without checking for traffic & rt and left turn & & \\
\hline & & 1 & questionable check for traffic during lane changes & steering & & \\
\hline & & 1 & switched turn lanes unsafely & rt and left turn & $x$ & $\mathbf{x}$ \\
\hline & & 1 & help with appropriate lane selection ---CUE & rt and left turn & $\mathbf{x}$ & \\
\hline & & 1 & required assistance for lane change ---ASSIST & highway driving & & $\mathbf{x}$ \\
\hline
\end{tabular}


The coding for indications of assistance applied the label CUE when words such as "help with...selection," "oriented," "cued," and "too...” were included in an error description. For the most part, the cues are forms of constructive criticism, but driving cues that point out the right of way or a nearby road hazard also reflected reduced levels of driving capability. The ASSIST label applied when a description included the word "assistance.” The error listings with a possibly-assisted event have the label $\mathrm{X}$ to show the potential extent of documented plus undocumented assistance for critical or unsafe errors that may also require cues or physical assistance while driving. The implied assistance (where CUE or ASSIST is labeled on a driving error) associated with low-frequency driving errors except for the top two errors classified under tracking.

In Table 2, the enumerated list of errors continued on to show documented concerns that occurred with turns and intersection negotiations. Once again, labels of CUE and ASSIST designated where steering assistance, braking assistance, and driving cues were explicitly used during on-road evaluation.

Table 2. Errors Associated with Driving on Road Crossings (Intersections)

\begin{tabular}{|c|c|c|c|c|c|c|}
\hline \multirow[b]{2}{*}{ Class } & \multirow[b]{2}{*}{ Total } & & \multicolumn{2}{|c|}{ Potential } \\
\hline & & Frequency & Errors & Context & CUE & ASSIST \\
\hline \multirow{9}{*}{ Turn } & \multirow{9}{*}{69} & 28 & over accel on left turns & rt and left turn & $\mathbf{x}$ & $\mathbf{x}$ \\
\hline & & 16 & inconsistent use of turn signal & signaling & & \\
\hline & & 13 & over shoot and under shoot & rt and left turn & $\mathbf{x}$ & $\mathbf{x}$ \\
\hline & & 5 & no mirror checks or unsatisfactory & rt and left turn & & \\
\hline & & 3 & awkward wheel recovery & steering & $\mathbf{x}$ & $\mathbf{x}$ \\
\hline & & 1 & oversteering & steering & $\mathbf{x}$ & $\mathbf{x}$ \\
\hline & & 1 & poor access of turn signals & signaling & & \\
\hline & & 1 & cues to turn signal off after turn ---CUE & signaling & $\mathbf{x}$ & \\
\hline & & 1 & nervous with quick actions & decision making & & \\
\hline \multirow{15}{*}{ Intersections } & \multirow{15}{*}{102} & $\overline{59}$ & rolled through stops & stopping & $\bar{x}$ & $\bar{x}$ \\
\hline & & 7 & hard acceleration & speed control & & \\
\hline & & 6 & insufficient opportunity to observe & traffic signs and signals & & \\
\hline & & 5 & trouble deciding right of way & traffic signs and signals & $\mathbf{x}$ & \\
\hline & & 4 & fast approach & intersections & $\mathbf{x}$ & $\mathbf{x}$ \\
\hline & & 3 & questionable check for traffic at intersections & rt and left turn & & \\
\hline & & 3 & long rolling stop towards stopped vehicles & intersections & & \\
\hline & & 3 & hard braking & stopping & & \\
\hline & & 2 & required assistance for late braking ---ASSIST & stopping & & $\mathbf{x}$ \\
\hline & & 2 & hesitant and slow & intersections & & \\
\hline & & 2 & missed stop sign & traffic signs and signals & $\mathbf{x}$ & $\mathbf{x}$ \\
\hline & & 2 & hesitant and stopped in middle of intersection & rt and left turn & $\mathbf{x}$ & \\
\hline & & 2 & cued to make more mirror checks ---CUE & intersections & $\mathbf{x}$ & \\
\hline & & 1 & overly cautious and slow stops & stopping & & \\
\hline & & 1 & missed waive through sign from driver yielding right of way & steering & $\mathbf{x}$ & \\
\hline
\end{tabular}

All clients of the ADP were viewed according to the outcome of their on-road evaluation and the ultimate recommendations reported in their case record. The secondary analysis excluded the records for clients needing adaptive equipment, because vehicle modifications require training and state testing for addition of a restriction to the driver's license. Below, Figure 1 presents the percentage of cases where a documented assistance was associated with passing or not passing (standard vehicle training or fail). In addition, the figure shows how documented and potential assistance relates to the percentage of detectable errors within each of the five classes defined in the prior tables. 


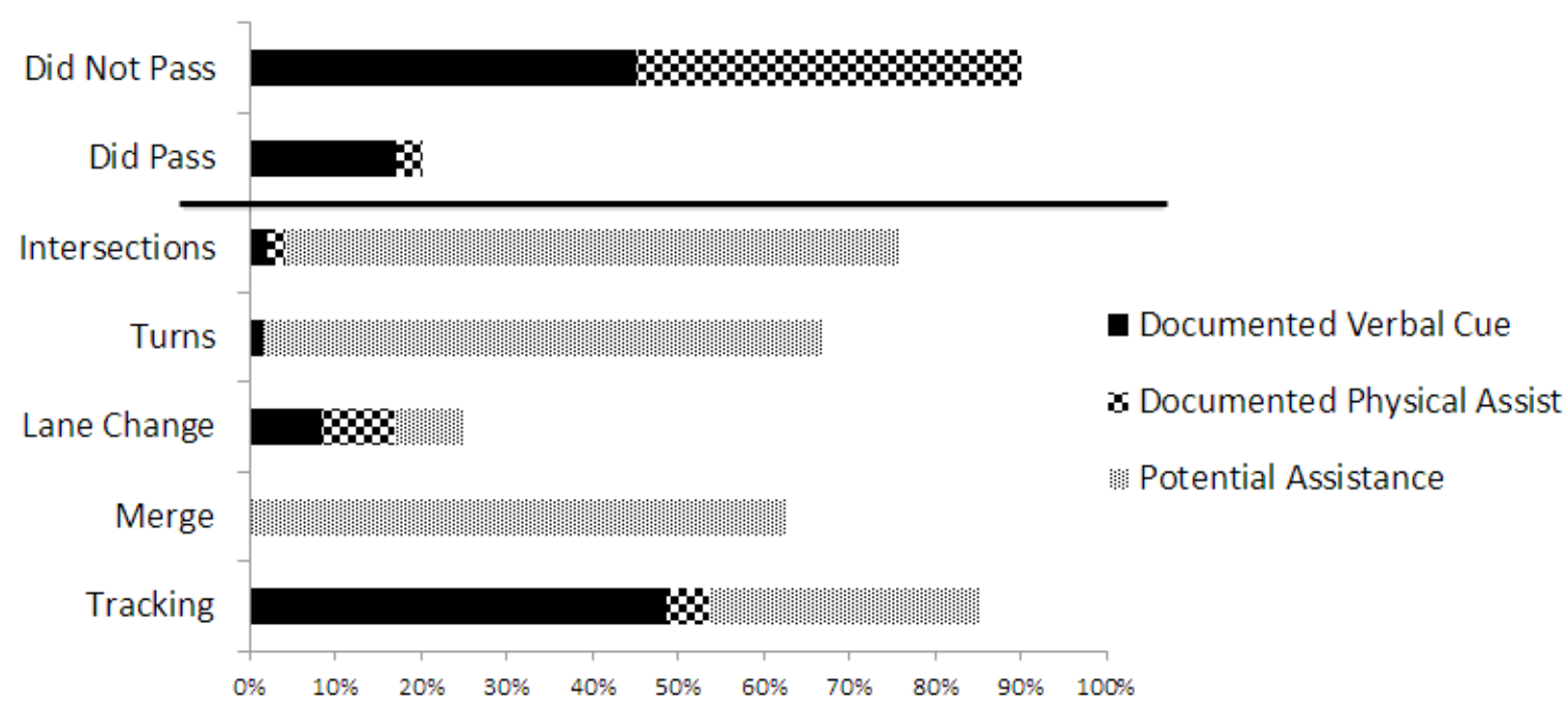

Figure 1. Percentages of Assistance Linked to Error Classes and On-Road Evaluation Outcomes

18 of the 20 clients who did not pass their on-road evaluation had an assisted-event (cues or assistance) reported in the findings of their baseline driving session. However, assisted-events were also documented in 14\% of the 58 clients who directly passed their on-road evaluation. Most of the detectable errors related to an assisted-event aligned with tracking within the lane of a road segment. This driving task of remaining "centered" within a lane has been reported in prior studies in driving assessment (Hoggarth, 2011) and driving simulation (Longhitano, 2012) as a critical source of driving errors. The association of steering errors has previously been associated with senior drivers in each of those prior studies.

While very few documented events of assistance occurred for driving maneuvers related to road crossings (intersections and turns), there was ample documentation of assistance for the driving maneuvers over road segments. There is no certainty whether these findings indicate that documentation accuracy declines with road crossings or if errors with road segment maneuvers are more significant when determining fitness to drive.

These findings presented a classification structure that may serve as a link from errors during driving evaluation to the contributing factors of at-risk motor vehicle collisions reported in police reports (Classen, 2010). Unique to this study, our comparison of assistance to class of errors also illustrates that explicitly documented assistance corresponded to relatively few of the total enumerated errors in reports. Though the errors may be few or less-frequent, assistance during supervised driving yields a specificity of 93.3\%. With the addition of "Potential Assistance" percentages, it is possible to make broader associations among errors related to steering or braking. The mapping of assistance to outcomes from baseline driving sessions provides significant support towards the advancement of a novel methodology called NAViSection (Beyene, 2011) using the measures reviewed in this study. 


\section{CONCLUSION}

Increasing demands for driving evaluations among people with medical impairments will challenge professionals to provide a greater level of counseling services when driving restrictions, training, or cessation are recommended. The availability of more evidence to support counseling and client education may promote greater trust and harmony among health care professionals and current or potential drivers. The results of this study illustrated that assistance by an evaluator during on-road evaluation were associated with $90 \%$ of the cases where clients did not pass their baseline driving session. While documented assistance mapped to a small percentage of the unique errors recorded within most classes, the simple nature of an error resulting in assistance appeared to turn low-frequency error events into high criticality ones in determination of a client's outcome.

Although the errors cannot be considered of equal weight to the demonstration of driver capability and determination of fitness to drive, the commission of errors that result in assistance could indicate a greater probability of crash involvement. These findings support our current development of in-vehicle technologies to digitally log assistance (steering assistance, braking assistance, driving cues) during on-road assessment. A future study will seek to quantify the frequency and duration of assistance in hopes of identifying a cut-point at which to differentiate assistance among clients who do not pass versus the clients who do pass.

\section{ACKNOWLEDGEMENTS}

This material is based upon work supported by the National Science Foundation under Cooperative Agreement EEC-0540865. Any opinions, findings, and conclusions or recommendations expressed in this material are those of the authors and do not necessarily reflect the views of the National Science Foundation. The contents do not represent the views of the Department of Veterans Affairs or the United States Government.

\section{REFERENCES}

Beyene, N. M., Lane, A., Seelman, K., Songer, T., Steinfeld, A., Cooper, R.A., (2011). Navisection: A Novel Method Joining Naturalistic Driving Data Collection with Expert Witness Event-logging for Enhanced Assessment of Driver Safety, 3rd International Conference on Road Safety and Simulation, Indianapolis IN, September 14-16.

Carr, D. B. (2010). Physician's guide to assessing and counseling older drivers. (2nd ed.). Chicago, IL: American Medical Association.

Classen, S., Shechtman, O., Awadzi, K. D., Joo, Y., \& Lanford, D. N. (2010). Traffic violations versus driving errors of older adults: informing clinical practice, American Journal of Occupational Therapy, 64(2), 233-241.

Crizzle, A., Myers, A., Vrklyan, B., \& Almeida, Q. (2011). Using In-Vehicle Devices to Examine Exposure and Patterns in Drivers with Parkinson's Disease Compared to an Age-Matched Control Group, Proceedings of the Fifth International Driving Symposium on Human Factors in Driver Assessment, Training and Vehicle Design, Lake Tahoe, CA, 263-269. 
Hoggarth, P., Innes, C., Dalrymple-Alford, J., \& Jones, R. (2011). On-Road Driving Assessment Errors Associated with Pass and Fail Outcomes for Older Drivers with Cognitive Impairment, Proceedings of the Fifth International Driving Symposium on Human Factors in Driver Assessment, Training and Vehicle Design, Lake Tahoe, CA, 351-357.

Jang, R. W., Man-Son-Hing, M., Molnar, F. J., Hogan, D. B., Marshall, S. C., Auger, J., Naglie, G. (2007). Family physicians' attitudes and practices regarding assessments of medical fitness to drive in older persons. [Comparative Study Research Support, Non-U.S. Gov't]. Journal of General Internal Medicine, 22(4), 531-543.

Longhitano, D. (2012). Translating Teen Driver Research from the Simulator to the Road. Proceedings of the Third International Symposium on Naturalistic Driving Studies, Blacksburg, Virginia, August 27, 2012.

Sheridan, T. B. (2008). Risk, human error, and system resilience: Fundamental ideas. Human Factors: The Journal of the Human Factors and Ergonomics Society, 50(3), 418-426. 